\title{
Question-answer pairs in Russian Sign Language: a corpus study
}

\author{
Evgeniia Khristoforova (ACLC, University of Amsterdam; \\ Russian State University for the Humanities) \\ evkhristoforova@gmail.com \\ Vadim Kimmelman (LLE, University of Bergen) \\ vadim.kimmelman@gmail.com
}

\begin{abstract}
We describe basic morphosyntactic and semantic properties of question-answer pairs (QAPs) collected from the online corpus of Russian Sign Language (RSL). We identified two classes of QAPs: classical and discourse QAPs, which are different in the semantic relation between the question and answer parts. We discovered that non-manual marking and word order in both types of QAPs are different from other constructions involving wh-signs, namely regular questions and free relative clauses. Guided by the similarity between non-manual marking of QAPs and role shift marking, we hypothesize on a possible grammaticalization process connecting the two constructions.
\end{abstract}

Keywords: question-answer pairs, wh-clefts, Russian Sign Language, grammaticalization, corpus research

\section{Introduction}

Many sign languages use a construction in which an apparent question is followed by an answer produced by the same signer, as in (1) from American Sign Language (ASL) (br: brow raise).

(1) JOHN BUY WHAT BOOK

'What John bought is a book.' (adapted from Caponigro and Davidson 2011, 329)

Such constructions have different names, also due to differences in analysis, as we will discuss in Section 2, but the most common term is wh-clefts (Wilbur 1996), emphasizing the parallel between this construction and pseudo-clefts in spoken languages. However, due to the presence of other analyses (and other similar constructions), we will use the neutral term question-answer pairs (QAPs), which does not presuppose a specific syntactic analysis.

QAPs have been described for several sign languages, and various analyses have been proposed (Section 2). In this paper, we present a corpus-based description of QAPs in another sign language, namely Russian Sign Language (RSL). We will argue that the properties of QAPs in this language add a valuable typological data point, as well as challenge some of the theories of QAPs proposed based on other sign languages.

Section 2 provides an overview of QAPs in other sign languages; Section 3 introduces the methods, and Section 4 the results. In Section 5 we discuss the significance of our findings. 


\section{Background}

QAPs have been described for a number of sign languages: ASL (Wilbur 1996; Caponigro and Davidson 2011), Sign Language of the Netherlands, NGT (Kimmelman and Vink 2017), Italian Sign Language, LIS (Branchini 2014), and French Sign Language, LSF (Hauser 2018), South African Sign Language, SASL (Huddlestone 2021); similar constructions have been mentioned to occur in other sign languages, too (e.g. Australian Sign Language, Johnston and Schembri 2007).

It is important to point out, however, that the properties of QAPs in different sign languages are not identical. Moreover, researchers disagree on what should and should not be analyzed as QAPs, or whether different types of QAPs should be offered a unified analysis within a single sign language.

For example, for ASL, different researchers have proposed competing analyses for these constructions. Wilbur (1996), in an influential study analyzed such constructions as pseudoclefts (also known as wh-clefts), adopting one of the existing analysis for the respective constructions in English. Under this approach, wh-clauses are predicative phrases, which raise to the left periphery of the sentence thus inducing subject-auxiliary inversion (see Heggie (1988) for details). An alternative analysis draws a parallel between wh-clause and free relative clauses introduced by an interrogative pronoun (Jespersen 1927). ${ }^{1}$

Caponigro and Davidson (2011) argued instead that QAPs in ASL are not wh-clefts, but question-answer clauses, where an embedded question clause is connected to an embedded answer clause by a null copula. Finally, Hoza et al. (1997) proposed an analysis for QAPs in ASL as a discourse-level phenomenon; basically they argued that QAPs are rhetorical question sentences followed by syntactically independent answer sentences.

The apparent disagreement between these researchers is largely not based on conflicting empirical observations. Instead, the main difference seems to be related to the scope of the proposed analyses and on whether the researchers pursue a unified analysis for different constructions. Thus, Hoza et al. (1997) developed an analysis that would include such question-answer sequences where there is a clear intervening clause between the question and the answer (2), as well as cases where the answer is only indirectly related to the question (wh: non-manual markers of wh-questions, namely brow frowns and head movements; neg: negative non-manual markers, that is, headshake). Thus, if such cases are to be accounted for by a single analysis for QAPs, QAPs have to be analyzed as a discourse-level phenomenon.

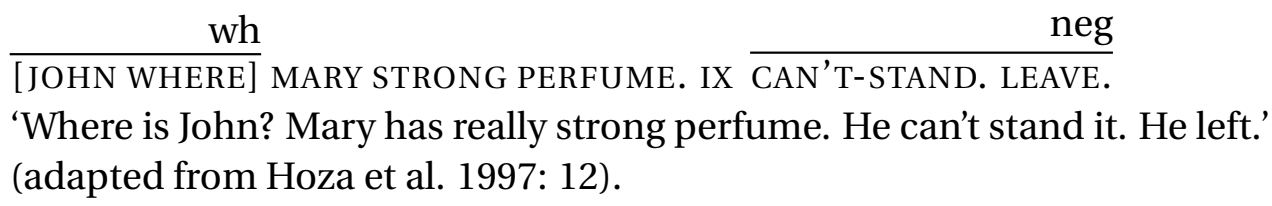

Both Wilbur (1996) and Caponigro and Davidson (2011) are aware of such cases, but they excluded them from the analysis that they develop, as the existence of discourse-level QAPs does not preclude the existence of syntactic QAPs as a separate construction.

Similarly, both Hoza et al. (1997) and Caponigro and Davidson (2011) include cases where the question part of the QAP is a polar question, as in (3) (y/n: non-manual markers of yes/no questions, namely brow raise and head tilt).

1. Relative clauses are dependent clauses which are connected to the main clause by a semantically and syntactically shared pivotal constituent, the head (Branchini 2014). Free relative clause (also known as headless relative clauses) is a subtype of relative clauses distinguished by a phonologically null head - e.g., I love what my mum cooks (ibid.). 
(3) $\overline{\text { IX } 1 \text { GO TOMORROW }} \frac{}{\text { NO, NEXT WEEK }}$

'Am I going tomorrow? No, next week.' (adapted from Hoza et al. 1997: 14).

Clearly, such cases cannot be analyzed as wh-clefts because they lack the wh-component. For Wilbur (1996), such cases, while attested, simply represent a construction which is structurally different from wh-clefts that she analyzed, and thus a wh-cleft analysis is still applicable to the cases containing wh-signs.

Please note that this overview of the differences between the analyses for QAPs in ASL is very brief and superficial. Consult the original sources for more details.

Kimmelman and Vink (2017), also followed by Hauser (2018), proposed a possible way of both acknowledging that the broad definition of QAPs includes constructions with different syntactic and semantic properties and unifying these different constructions. For example, in NGT corpus data, one can find QAPs which are clearly syntactic (e.g. cases where the whole QAP is embedded as a complex of a matrix predicate), and QAPs which are clearly discourse-level (as in (2)). The solution is to say that this is a result of ongoing grammaticalization of a syntactic construction from a discourse-level sequence of sentences. Thus, syntactic QAPs and discourse-level QAPs are different constructions, but they are diachronically related. Hauser (2018) argued that it is possible that some QAPs in different sign languages have grammaticalized to different extents: while QAPs in NGT (as analyzed by Kimmelman and Vink 2017) do not have properties of wh-clefts, in LSF they are on the way but not yet analyzable as wh-clefts, while in LIS (as analyzed by Branchini 2014), they are in fact wh-clefts.

While a grammaticalization analysis of QAPs is attractive, some notes of caution are due. First, even if it is true that some QAPs in a language have grammaticalized from other QAPs, it is still necessary to carefully analyze morphosyntactic properties of these constructions in order to probe deeper into the mechanisms underlying the language change and, also, enrich the typology of these constructions. Second, such grammaticalization accounts are by definition speculative, as for most if not all sign languages, we do not have diachronic records that would allow for actually reflecting grammaticalization. ${ }^{2}$ Third, note that grammaticalization should not be used as a value judgement, as, for example, to argue that some languages are more "developed" than others if they have grammaticalized some construction that others have not. Finally, as we will demonstrate in this paper, QAPs in RSL might present some specific empirical challenges to grammaticalization accounts.

\section{Data and Methods}

\subsection{The corpus}

In this study, we used the freely-available online RSL corpus (Burkova 2015). The corpus contains approximately 200 video clips collected from 43 deaf RSL signers, who mostly come

2. One of the methods to explore diachronic changes without historical record is to compare constructions produced by different generations of signers (see Dachkovsky 2018, 2020). Although this method may provide some insights into the sociolinguistic variation in the usage of QAPs, we do not expect it to immediately uncover the diachronic processes behind the development of this construction. This is because the method relies on the premise that age-related language differences reflect language change alone. However, there are numerous other factors that can eventually result in generation difference (e.g., changes in education and language policies). Furthermore, given that RSL is around 200 years old, we might not see a lot of diachronic change by comparing different generations of signers currently using RSL: presumably, the most rapid change occurs in the early decennia of the existence of a sign language. 
from two large Russian cities, Moscow and Novosibirsk. Background information (age, family status, city of birth, etc.) can be found on the website of the corpus. The recordings represent different text types (picture-based storytelling, interviews, spontaneous narrations, etc.) and are annotated in ELAN software (https://tla.mpi.nl/tools/tla-tools/elan/). The corpus annotations contain separate tiers for sign glosses for the right and the left hands, sentenceby-sentence translations, and comments.

\subsection{Query strategies and ambiguous cases}

In order to identify QAPs in the corpus, we searched for wh-signs on the gloss tier and further manually analyzed the output. Because we did not have any null hypothesis of how QAPs are marked in RSL, we had to rely primarily on the contextual cues. Our working definition of QAPs was that it is a construction consisting of two component, namely a question followed by an answer, where both components are authored by the same person. Note, that in the beginning we deliberately ignored such potentially valuable cues as non-manual marking associated with regular questions and/or particular information structure. As we show further, this proved to be empirically justified. The above-mentioned definition allowed us to exclude constructed dialogues, where question and answer may both be uttered by the same signer, but, once examined in the context of the whole narration, appeared to have different authors.

Another source of potential ambiguity are non-interrogative uses of wh-signs in RSL. For instance, indefinite pronouns SOMEONE and SOMETHING in RSL are homonyms with interrogative signs WHO and WHAT, respectively. Sometimes, indefinite pronouns and interrogatives are distinguished by mouthing (i.e., silent mouth gestures mimicking articulation of corresponding Russian words). However, mouthing is optional for both elements and, therefore, is not a reliable cue (see Kimmelman 2018 for more discussion on indefinite pronouns in RSL). The homonymy between indefinite pronouns and interrogatives is problematic primarily in those cases where a construction in focus is additionally ambiguous between mono- and bi-clausal readings. Example (4) illustrates such an ambiguous case, which was excluded from the analysis along with similar examples.

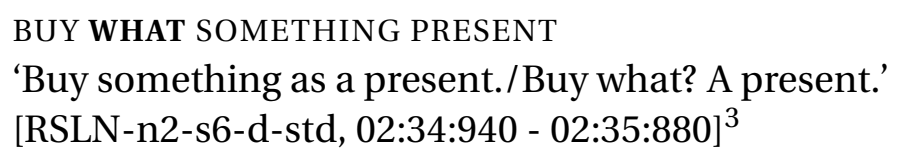

In addition, the sign WHAT can also serve as a complementizer introducing a complement clause (5). Again, neither non-manual markers nor the form of the sign can help to identify the function of WHAT in isolation. An example of an ambiguous use of WHAT is given in (5). This type of examples was also excluded from our pool.

\section{GLAD WHAT REVENGE}

'He is glad that he payed back. / He is glad about what? Paying back.' [RSLN-b3-s4-d-std, 01:12:760 - 01:14:900]

Finally, we found that interrogative signs can be used to introduce free relative clauses. We will come back to free relative clauses in Section 4. For now, it suffices to illustrate how

3. The square brackets in the end of the RSL examples contain the name of the respective video clip in the online RSL corpus and the time-codes. The name of the clip is also a link to the relevant file in the corpus. Note that free registration is required to view the data. 
we disambiguated free relatives and QAPs in our analysis relying primarily on their surface syntactic and semantic properties. Thus, free relative clauses occupy the argument position in the clause, while QAPs under any analysis presented in Section 2 do not. Consider the following example (6) (eb: eye blink):

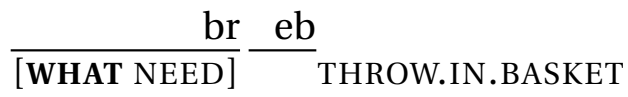

Lit., 'What I need, I throw in the basket.'

'I throw in the basket what is needed.'

[RSLN-b20-s30-d-std, 00:20:615 - 00:22:165]

It is evident that the second clause in (6), THROW.IN.BASKET, under no interpretation contains an answer to the alleged question in the first clause, WHAT NEED. Instead, the latter modifies the focused null object of the second clause, potentially answering the question of what the signer throws into the basket. Whenever the interrogative clause could be analysed as an argument of the non-interrogative clause as in (6), the construction was regarded as a free relative clause and thus excluded from the analysis.

\subsection{Annotation}

In order to pursue any analysis of QAPs, it is necessary to describe their basic morphosyntactic and semantic properties; furthermore, their properties should be compared to the properties of other related constructions, such as regular questions and free relative clauses. ${ }^{4}$

Based on the previous studies of QAPs in other sign languages discussed in Section 2, we decided to analyze a number of morphosyntactic and semantic properties of QAPs in RSL. For each QAP identified in the corpus, we described the following parameters:

- the wh-sign involved

- the position of the wh-sign in the question part

- the constituent order in the question and answer parts

- the type of the question part: discourse/classic (see explanation below)

- the type of the answer part: direct/indirect; full/reduced (see explanation below)

- the non-manual markers on both parts

In addition to QAPs, we searched for regular questions introduced by the same interrogative signs. This was necessary to provide the comparison for the question component of QAPs to investigate whether regular questions and question parts of QAPs are different in terms of morphosyntactic marking (e.g., non-manual markers, constituent order, etc.). In contrast with QAPs, questions were identified as regular interrogative sentences that were not followed by the answer authored by the same person.

4. The comparison to free relative clauses is necessary because, as argued by Caponigro and Davidson (2011), wh-clefts in spoken languages have free-relative clauses as their wh-components. Thus, to pursue a wh-cleft analysis, it is necessary to test whether the question part of the QAP can be analyzed as a free relative clause. However, different syntactic analyses of wh-clefts exist (Wilbur 1996), so this is not a fully decisive argument. 


\section{Results}

In total, the corpus yielded 53 QAPs, 70 regular wh-questions and 8 free relatives introduced by interrogative signs. Let us first focus on the QAPs. Note that all wh-signs that are used in regular questions in the corpus are also used in QAPs, except for WHERE and WHAT.FOR (which is likely to be related to the small corpus size).

Based on the analysis of the structure of question and answer parts of QAPs found in the corpus, it was possible to divide them into two classes, which we call "classical QAPs" and "discourse QAPs", which are described in Sections 4.1 and 4.2 respectively. We show that the two types of QAPs differ compositionally (i.e., the relation between the question and answer parts differs between two types of QAPs), but their morphosyntactic marking (non-manual making and constituent order) remains the same for both types (Section 4.3).

\subsection{Classical QAP}

Classical QAPs receive their label because their structure accurately patterns together with QAPs in other sign languages, such as ASL described in Wilbur (1996). Thus, the question part of classical QAPs consists of a full interrogative clause, while the answer part contains a focused demonstrative phrase (DP), which directly answers the question posed in the question part. Consider example (7) (blr: body lean right).

(7) $\frac{\text { gaze }}{\text { FOLLOW wHo DOG ALIEN FAT }}$
'Who follows? A fat alien dog.'
[RSLN-b17-s28-h-std, 00:11:480 - 00:14:590]

The question part of QAP in (7), FOLLOW WHO, is a full interrogative clause, while the answer part, DOG ALIEN FAT, contains only a focused DP which matches the category of the respective wh-sign in the question part. Thus, for classical QAPs it is possible to suggest a syntactic approach treating QAPs as complex sentences (either following the wh-cleft analysis in Wilbur 1996 or the null copula analysis in Caponigro and Davidson 2011). However, QAPs of this kind constitute less than a half of the sample (38\%; 20 entries). The position of the wh-sign and non-manual marking of classical QAPs are addressed in Section 4.3.

\subsection{Discourse QAP}

The rest of the QAPs found in the corpus (62\%; 33 entries) have a different structure. Specifically, the answer part in these constructions does not directly answer the question in the question part, but rather consist of a full declarative clause. Thus, in example (8), a clause in the answer part of the QAP, NEED RESCUE, does not answer the question DO WHAT? (the proper answer would be just RESCUE).

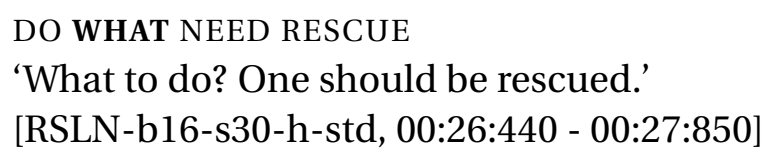

Still, the answer part in (8) makes at least pragmatic sense since it contains the target answer, albeit not limited to it as in the classical QAP in (7). This is, however, not always the case. Consider example (9) (CL(): size and shape classifier): 
(9) CL(RECTANGLE) WHICH KNOW.NOT

'What is this rectangular thing? (I) don't know.'

[RSLN-n3-s2-d-std, 01:57:855 - 01:59:795]

The answer part in (9) does not contain a constituent, that would match the category of the wh-sign in the question part. Nevertheless, we will show that this sentence conforms to the morphosyntactic marking of QAP along with examples (7-8) discussed so far.

Finally, there is a subtype of discourse QAP, which exhibits yet another functional property. In this type, the question part is reduced to a wh-sign only, while the answer part remains a full clause as in (10) (bf: brow frown).

(10) WHAT $\frac{\mathrm{br}}{\text { WALK }}$ PEOPLE WALK P-A-R-K

'People are walking in the park.'

[RSLN-n5-s2-d-std, 02:37:815 - 02:41:705]

Interestingly, the construction in (10) occurs in the very beginning of the narrative thus setting the scene for the following discourse. Note that wh-clefts are generally used to separate focus from presupposed information, which contrasts with this use of QAPs where no presupposition is present.

\subsection{Morphosyntactic marking of QAPs in RSL}

As mentioned above, the different types of QAPs in RSL do not differ from each other in terms of non-manual marking and constituent order. Both, however, do differ from other constructions involving wh-signs, such as questions and free relative clauses.

\subsubsection{Non-manual markers}

Non-manual marking of QAPs in RSL maps on their internal structure in such a way that the question and answer parts of QAPs are marked separately. The distribution of non-manual markers (NMMs) across different parts of QAPs is given in Table 1. Apart from the scope of non-manual markers specific to the question and answer parts, the QAP-internal clause boundary can also be marked by an eye blink.

\begin{tabular}{lll}
\hline Question part NMMs & Marked QAPs & $\begin{array}{l}\text { \% of QAPs } \\
\text { (53 in total) }\end{array}$ \\
\hline gaze & 21 & $40 \%$ \\
lateral head/body movements & 18 & $34 \%$ \\
\hline Answer part NMMs & & \\
\hline head lean forward & 17 & $32 \%$ \\
lateral head/body movements & 8 & $15 \%$ \\
\hline QAP-internal clause boundary (eye blinks) & 19 & $36 \%$ \\
\hline
\end{tabular}

Table 1: Non-manual marking of QAPs in RSL

Table 1 shows that the non-manual marking of QAP is rather inconsistent since none of the non-manual markers is attested in more than half of the constructions. Importantly, however, each QAP in the sample has at least one of the non-manual markers listed in Table 1. 
Let us first consider the marking of the question part. Intuitively, one may expect the non-manual marking of the question part to resemble the respective marking of regular questions. However, this is not the case in RSL. As Table 1 illustrates, the question parts of QAPs are frequently marked with side-ward eye gaze and lateral body and head movements. This set of non-manuals is not typically associated with questions (see Table 2 below) but is reminiscent of another well-attested sign language phenomenon, namely role shift (Kimmelman and Khristoforova 2018). Role shift non-manual marking implies shifting into the role of the person whose speech, thought, emotions or actions are reported. It is often expressed as a lateral body and head movements in combination with side-ward eye gaze. At the same time, in the context of QAP, there is nobody in the discourse whose role can be assumed (otherwise, the construction would be identified as a reported dialogue and excluded from the analysis according to the criteria in Section 3.2). The connection between role-shift and QAP is addressed in Section 5.

In order to illustrate that the question part lacks canonical non-manual markers of questions, we analyzed a sample of 70 regular questions in the corpus. Within this sample, we identified the following non-manual markers:

\begin{tabular}{lll}
\hline NMMs of questions & Marked questions & $\begin{array}{l}\text { \% of questions } \\
\text { (70 in total) }\end{array}$ \\
\hline brow raise & 20 & $29 \%$ \\
brow frown & 23 & $33 \%$ \\
head lean back & 30 & $43 \%$ \\
chin forward & 8 & $11 \%$ \\
\hline
\end{tabular}

Table 2: Non-manual marking of regular questions

Table 2 demonstrates that questions in our sample are marked with specific brow patterns, backward head movements and chin fronting. This is in line with what is observed in other sign languages (see Cecchetto (2012) for an overview). However, none of this non-manual markers is observed in the question part of QAPs in sufficient amounts, which allows us to conclude that QAPs cannot be regarded as regular discourse-level question-answer sequences. $^{5}$

Let us now turn to the answer part. Table 1 reveals that the answer part is also marked rather inconsistently. The most frequent non-manual marker of the answer part - i.e., the forward head lean - scopes either over the whole answer part or over the focus constituent only in discourse QAPs. This marking relates to the information structure associated with QAP constructions, which is the separation of the focus from the presupposed information. As for lateral body and head movements, these serve to counterbalance the respective movement in the question part therefore visually marking an opposition between two clauses.

Although in general none of the markers is consistently present in the majority of QAPs, some non-manual marking is always present thereby contrasting the two parts of QAPs (either by different non-manual markers of by marking only one of the parts of the construction). Apart from contrasting the marking of the question and answer parts, the boundary between the two can also be marked by an eye blink. Contrary to what might be expected,

5. As for the non-manual marking of free relatives introduced by wh-signs, it was not systematically investigated due to the insufficient number of corpus entries. However, in Khristoforova and Kimmelman (2020), we examine other types of relative clauses (including free relatives introduced by non-interrogative pronouns), which exhibit patterns of non-manual marking different from what we observe in QAP. 
there is no perceptually observable/measurable pause between the clauses within QAPs.

\subsubsection{Position of the wh-sign}

Another point of attention is the position of the wh-sign in the QAPs. As in case of nonmanual marking described above, different types of QAPs do not diverge from each other in this parameter but do differ from other constructions involving wh-signs. Table 3 demonstrates that wh-signs in QAP are predominantly on the right periphery of the question part, that is, on the boundary between the two clauses. This is also true for wh-signs whose position is marked as "single" (i.e., with the question part consisting of a wh-sign only) or "doubled" (i.e. wh-sign is present in both the initial and the final positions in the clause). Importantly, the position of the wh-sign in QAP is not influenced by the category of the wh-sign or its base-generated position. ${ }^{6}$

\begin{tabular}{lclllll}
\hline Position of the wh-sign & final & initial & single & doubled & center & total \\
\hline QAP (Q-part) & 37 & 5 & 8 & 2 & 1 & 53 \\
Questions & 14 & 25 & 25 & 5 & 1 & 70 \\
Free relatives & 2 & 6 & & & & 8 \\
\hline
\end{tabular}

Table 3: Position of the wh-sign in the question part of the QAP

The distribution of wh-signs in regular questions vividly illustrates that these are structured in a different way. Disregarding questions consisting of a wh-sign only, the rest of the sample shows a weak tendency towards initial position of the wh-sign, while the final position as well as doubling are also widely attested. Note, that, as in the case of QAPs, the surface position of the wh-sign does not strongly correlate with its base-generated position. Thus, the sign WHAT, which is by far the most frequent in our sample of regular questions (32 entries) always marks the left periphery of the question regardless its syntactic function. Example (11) illustrates a question introduced by a sentence-initial wh-sign (bhl: backward head lean).

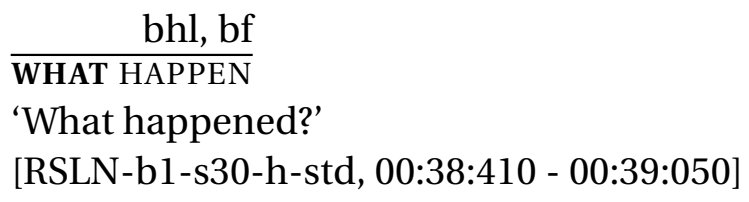

As for the free relatives identified in our sample, albeit few in number, these exhibit an even stronger tendency for initial wh-signs (see example (6)). In sum, we can conclude that the underlying structure of QAPs is different from those of free relatives and regular questions despite the use of the same inventory of functional elements, i.e. wh-signs.

\section{Discussion}

The properties of QAPs in RSL are interesting in that they seem to be different from QAPs in other sign languages in some respects, and also in that they demonstrate variability that is similar to other sign languages.

Concerning the similarities between RSL and other sign languages, several points have to be made. First, question parts of QAPs in RSL are clearly morphosyntactically different from

6. Due to the small size of the dataset, this observation is mainly based on analyzing WHAT and WHO. 
both regular questions and free relative clauses. In contrast to these other constructions, question parts of QAPs prefer the clause-final position of the wh-sign. Furthermore, the nonmanual marking is different in these three constructions.

Since the question parts of QAPs are different from regular questions, it is reasonable to argue that QAPs are not simply sequences of questions and answers in discourse. Note, however, that we did not investigate specifically the properties of rhetorical (non-information seeking questions) in RSL, and thus this analysis cannot be completely excluded. The fact that question parts of QAPs are different from free relative clauses can be used as an argument against a wh-cleft analysis of QAPs in RSL. However, this depends on accepting the argument that wh-clefts have to contain free relative clauses (Caponigro and Davidson 2011, but see Wilbur (1996) and Branchini (2014) for an alternative view).

Turning to the differences between RSL and other sign languages, it seems that the specific non-manual marking of QAPs in RSL is different from what has been described in the literature. The marking we found resembles more that of role shift, even though it is not reasonable to assume the presence of role shift in these examples.

Another important feature of QAPs in RSL concerns the two main classes of QAPs, which we identify on semantic/pragmatic grounds, namely classical and discourse QAPs. While these two types of QAPs are clearly different in terms of the relation between the question and answer parts, morphosyntactically we do not find differences between them.

This latter observation is important for the possible grammaticalization account of QAPs in RSL. It might seem attractive to argue that classical QAPs in RSL have grammaticalized from discourse QAPs. However, if this is the case, then this grammaticalization process only concerns semantics/pragmatics, but not morphosyntax or prosody, which seems to go against the definition of grammaticalization as "the creation of new functional material" (Roberts and Roussou 2003, 2). At best, the observed change can be the first step of a prospective grammaticalization process, where the semantic/functional change may probably lead to a formal change in future.

At the same time, we would like to propose that grammaticalization might be involved in the domain of QAPs, albeit not in relation to the two types of QAPs. As mentioned above, in many instances of QAPs we find non-manual markers similar to those of role shift: side-ward eye gaze and head and body movements. It is thus conceivable that QAPs in RSL originate not from simple questions, or sequences of simple rhetorical questions followed by answers, but from constructed dialogue sequences, where the signer takes on the role of one person asking the question and another person answering it. For questions in constructed dialogue, we have checked the corpus of examples collected for a different study of quotation in RSL (Kimmelman and Khristoforova 2018 and found that questions in constructed dialogues pattern together with regular questions and not with QAPs in terms of non-manual marking. Specifically, they are most commonly marked by eyebrow raise and head tilts. In addition, as expected, questions in constructed dialogue are marked by role shift non-manual markers, such as head and body turns and eye gaze.

This grammaticalization account seems to be more plausible for the QAPs in RSL because both the semantic/pragmatic and the formal properties of the construction under grammaticalization are affected. The source construction (constructed dialogue) involves non-manual markers of role shift and those typically associated with regular questions, the constituent order associated with regular questions, and the semantics/pragmatics of two people exchanging information. The target construction of QAPs generally preserves the role shift non-manuals, but loses the question non-manuals, and modifies the constituent order. Semantically and pragmatically, there is a clear change from constructed dialogue to 
highlighting important information in discourse.

Finally, recall that, as we discussed in Section 2, grammaticalization accounts including the one we just presented, are speculative, as it is difficult or impossible to actually test them. Thus, although it is tempting to regard grammaticalization as a smooth unidirectional transition from more to less complex structures, this need not always be the case (cf., Fischer 2013) and therefore should be empirically justified. Furthermore, our study is limited due to being based on corpus data only. Because of this, we analyzed a small set of QAPs and regular questions, and did not have access to negative data (i.e, examples of ungrammatical QAPs). This means, among other, that we do not have clear instances of embedded QAPs (but we also cannot claim that embedding is impossible), nor do we have clear examples of polar questions in QAPs (with the same caveat). We intend to further investigate morphosyntactic and semantic properties of QAPs in RSL using elicitation in future research.

\section{Acknowledgments}

We thank the audience of FEAST 2021 for their comments, and especially Ronnie Wilbur for her detailed feedback on our presentation, and Felix Sze for her comments on the manuscript. This research has been funded by the RSF grant 17-18-01184.

\section{References}

Branchini, Chiara. 2014. On relativization and clefting: an analysis of Italian Sign Language. Boston: De Gruyter Mouton ; Ishara Press.

Burkova, Svetlana. 2015. Russian Sign Language Corpus [Electronic resource]. http://rsl.nstu. $\mathrm{ru} /$.

Caponigro, Ivano, and Kathryn Davidson. 2011. "Ask, and tell as well: Question-Answer Clauses in American Sign Language.” Natural Language Semantics 19 (4): 323-371. https:/ / doi. org/10.1007/s11050-011-9071-0.

Cecchetto, Carlo. 2012. “Sentence types.” In Sign language: An international handbook, edited by Roland Pfau, Markus Steinbach, and Bencie Woll, 292-315. Berlin/Boston: De Gruyter Mouton.

Dachkovsky, Svetlana. 2018. "Grammaticalization of intonation in Israeli Sign Language: From information structure to relative clause relations." PHD Dissertation, University of Haifa.

. 2020. "From a demonstrative to a relative clause marker: Grammaticalization of pointing signs in Israeli Sign Language.” Sign Language \& Linguistics 23 (1-2): 142-170. https: //doi.org/10.1075/sll.00047.dac.

Fischer, Olga. 2013. "An inquiry into unidirectionality as a foundational element of grammaticalization: On the role played by analogy and the synchronic grammar system in processes of language change." Studies in Language 37 (3): 515-533. https:// doi.org/10. 1075/sl.37.3.03fis.

Hauser, Charlotte. 2018. "Question-answer pairs: the help of LSF." FEAST. Formal and Experimental Advances in Sign language Theory 2. https://doi.org/10.31009/FEAST.i2.04. 
Heggie, Lorie. 1988. “The syntax of copular structures.” Doctoral Dissertation, University of Southern California.

Hoza, Jack, Carol Neidle, Dawn MacLaughlin, Judy Kegl, and Ben Bahan. 1997. "A unified syntactic account of rhetorical questions in American Sign Language.” In Syntactic structure and discourse function: an examination of two constructions in American Sign Language, edited by Carol Neidle, Dawn MacLaughlin, and Robert G. Lee, 1-23. Boston: ASLLRP publications.

Huddlestone, Kate. 2021. "Negation and polar question-answer clauses in South African Sign Language.” Sign Language \& Linguistics 24 (1): 63-86. https://doi.org/10.1075/sll.19014. hud.

Jespersen, Otto. 1927. A modern English grammar III. London: George Allen \& Unwin.

Johnston, Trevor A., and Adam Schembri. 2007. Australian sign language (Auslan): An introduction to sign language linguistics. Cambridge, UK ; New York: Cambridge University Press.

Khristoforova, Evgeniia, and Vadim Kimmelman. 2020. "Relativization in Russian Sign Language: basic features.” Voprosy Jazykoznanija 6:48-65.

Kimmelman, Vadim. 2018. "Impersonal reference in Russian Sign Language (RSL).” Sign Language \& Linguistics 21 (2): 204-231. https:// doi.org/10.1075/sll.00018.kim.

Kimmelman, Vadim, and Evgeniia Khristoforova. 2018. "Quotation in Russian Sign Language: insights from corpus and elicitation." FEAST. Formal and Experimental Advances in Sign language Theory 2. https://doi.org/10.31009/FEAST.i2.08.

Kimmelman, Vadim, and Lianne Vink. 2017. "Question-Answer Pairs in Sign Language of the Netherlands.” Sign Language Studies 17 (4): 417-449. https:/ / doi.org/ 10.1353/sls.2017. 0013 .

Roberts, Ian, and Anna Roussou. 2003. Syntactic Change: A Minimalist Approach to Grammaticalization. Cambridge University Press. Cambridge.

Wilbur, Ronnie B. 1996. "Evidence for the function and structure of wh-clefts in American Sign Language.” International review of sign linguistics 1:209-256. 